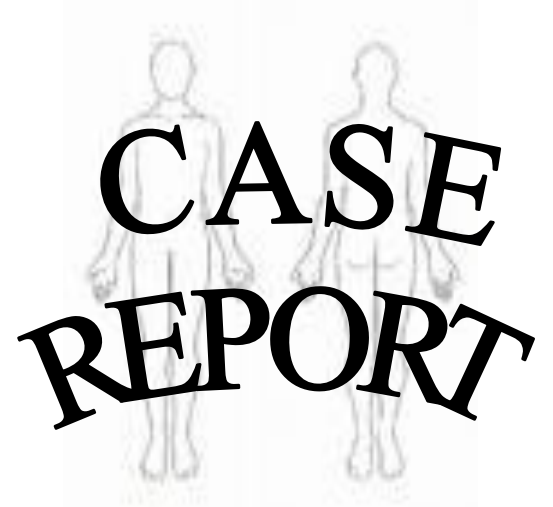

\title{
Atrial Flutter Converted to Sinus Rhythm by Adenosine Infusion
}

Furqan S Siddiqi, M.D. ${ }^{1}$, Adeel-ur-Rahman Siddiqui, M.D. ${ }^{2}$, Rami Jambeih, M.D. ${ }^{3}$, Wassim Shaheen, M.D. ${ }^{4,5}$

${ }^{1}$ University of Florida College of Medicine, Jacksonville, FL

${ }^{2}$ Tabba Heart Institute, Karachi, Pakistan

${ }^{3}$ University of Oklahoma Health Sciences Center, Oklahoma City, OK

${ }^{4}$ Heartland Cardiology, Wichita, KS

${ }^{5}$ University of Kansas School of Medicine-Wichita, KS

\section{Introduction}

Atrial flutter (AF) is the reentrance of signals in the atria resulting in beating up to five times faster than normal (i.e., 240-400 bpm). ${ }^{1}$ Common symptoms experienced during an episode of atrial flutter includes palpitations, dizziness, chest tightness, shortness of breath, and fatigue. To manage atrial flutter, four major concerns must be addressed: (1) reversion to normal sinus rhythm (NSR), (2) maintenance of NSR, (3) control of the ventricular rate, and (4) prevention of systemic embolization. Antiarrhythmic (AA) medications can be used to either "pharmacologically convert" a person to NSR or to maintain it following an external electrical cardioversion. ${ }^{2}$

Several medications are available for the treatment of atrial flutter including diltiazem, digoxin, and amiodarone. Adenosine, which is used for the treatment of paroxysmal supraventricular tachycardia (PSVT), is not indicated for the treatment of AF primarily due to its very short half-life.

We present a case of atrial flutter with rapid ventricular response, which was converted to NSR by administering a single dose of adenosine in a patient who underwent a surgical resection of cardiac leiomyosarcoma.

\section{Case Report}

A 51-year-old Caucasian male presented with abdominal pain and palpitations. On electrocardiogram, he had atrial fibrillation with a heart rate of $90 \mathrm{bpm}$. There was evidence of a large right atrial mass on 2-D echocardiogram. He was started on diltiazem infusion and rapidly converted to NSR. He also had tamponade and underwent pericardiocentesis successfully.

The patient was discharged home on diltiazem $120 \mathrm{mg}$ daily then later readmitted to the hospital for surgical removal of the cardiac mass. The pathology was positive for leiomyosarcoma. Postoperatively (Day 0 ), he developed AF and was treated with amiodarone. On post-operative day 1 , he developed another episode of rapid AF which was treated with digoxin. The next day, he had a third episode of AF with a rate of $114 \mathrm{bpm}$ (Figure 1). The electrocardiogram was misinterpreted as supraventricular tachycardia rather than atrial flutter with atrioventricular (AV) block. Adenosine $12 \mathrm{mg}$ was administered intravenously resulting in atrioventricular block (Figure 2) followed by rapid conversion to NSR. An electrocardiogram taken about 15 minutes after adenosine infusion showed a NSR (Figure 3). 


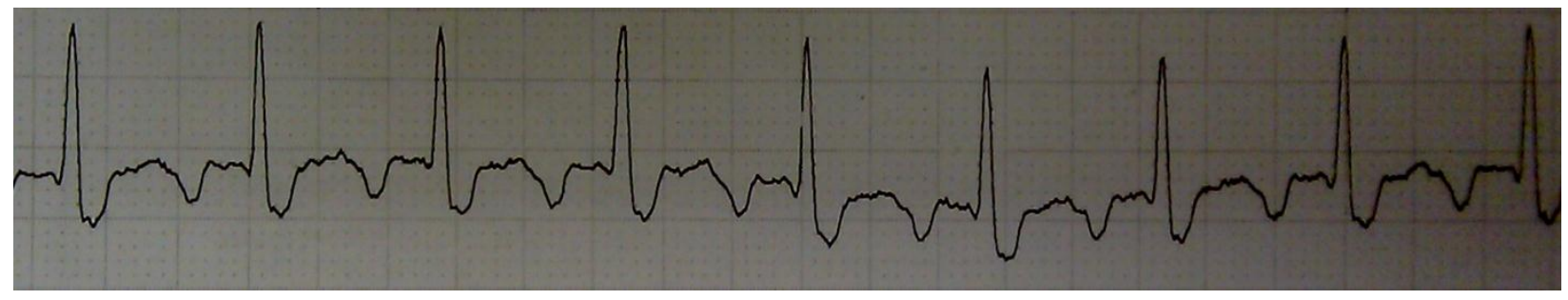

Figure 1. Atrial flutter with a rapid ventricular response.

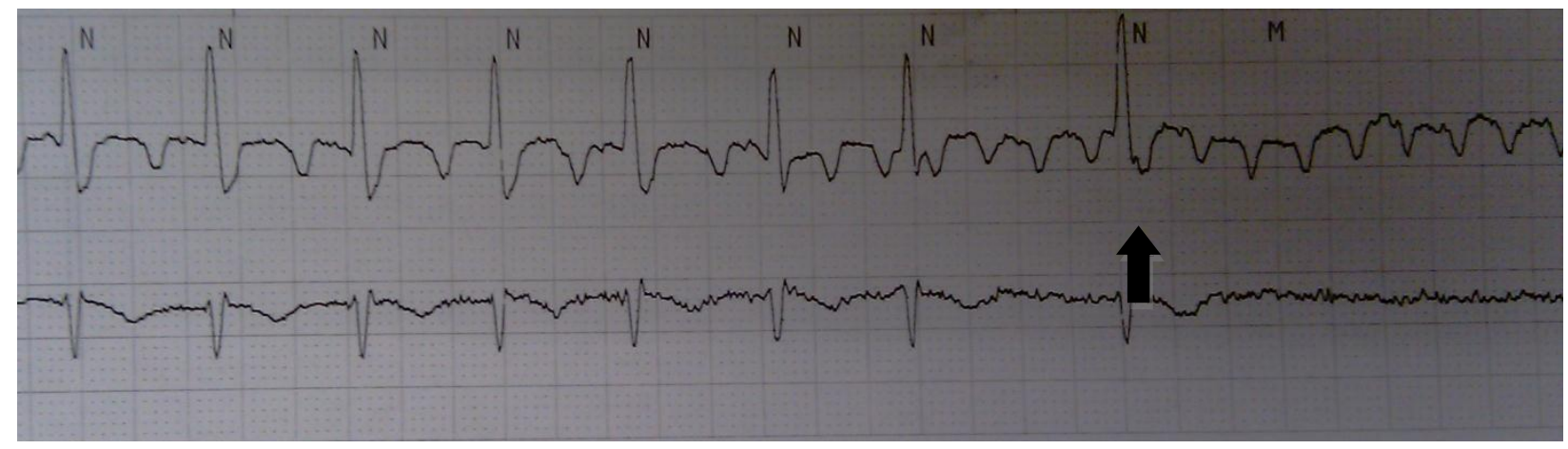

Figure 2. Atrial flutter/fibrillation shown with the administration of adenosine (black arrow).

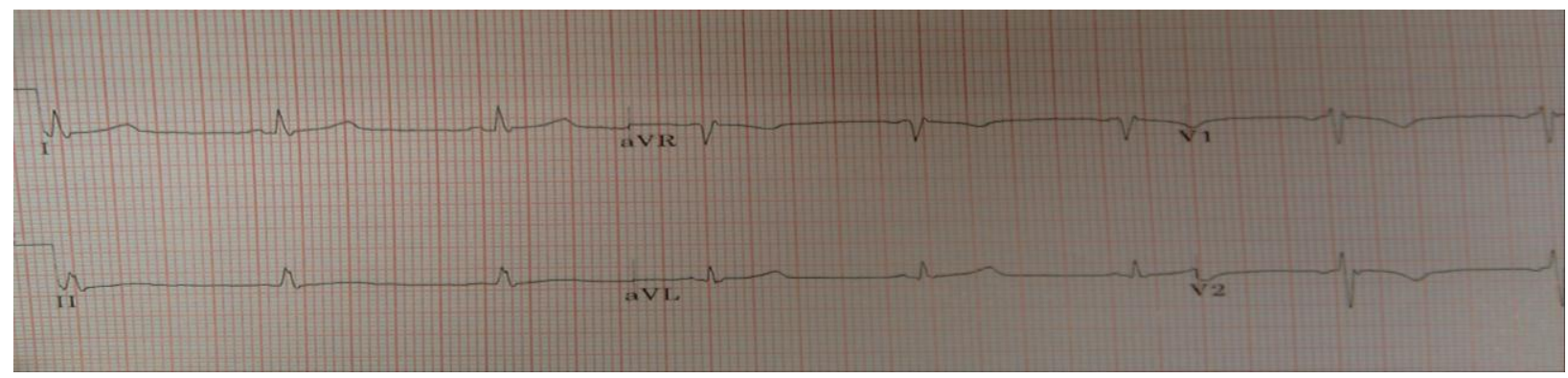

Figure 3. Normal sinus rhythm shown three hours after administration of adenosine.

\section{Discussion}

Adenosine is a potent chemical which has cardiac electrophysiologic effects including a negative chronotropic action on the sinus node and a predominant negative dromotropic action on the AV node. ${ }^{3}$ The negative dromotropic action has led mainly to the use of adenosine as a potent antiarrhythmic agent for paroxysmal supraventricular tachycardia (PSVT) mediated by a reentrant mechanism involving the AV node. The effects of adenosine are dose-dependent and of very short duration since the half-life is less than
10 seconds. ${ }^{4}$ This effect likely is related to the activation of the autonomic nervous system, mainly sympathetic, that occurs after administration of adenosine. Due to the short half-life, the sympathetic effects of adenosine are short-lived. The onset of 1:1 conduction most likely further increases sympathetic stimulation, thereby preventing return to 2:1 atrioventricular nodal conduction. $^{5}$

There is limited reported experience of the use of adenosine in patients with atrial arrhythmias and 2:1 AV block. In one study, 
adenosine administered to patients with atrial flutter and 2:1 block was not associated with acceleration of ventricular rate. ${ }^{5}$ No adverse effects were noted when adenosine was administered to 14 patients with intra-atrial reentrant tachycardia, though the number of patients who received the drug in the presence of 2:1 atrioventricular nodal block was not reported. ${ }^{6}$ These studies together with the fact that rhythm acceleration was not seen when adenosine was given to eight patients with atrial flutter and 2:1 conduction block through an accessory atrioventricular connection (where there are no counteracting effects of adenosine-induced conduction block) suggested that the effect of adenosine-induced sympathetic stimulation is likely to be small. ${ }^{6,7}$ In this case, the fact that it was sufficient to allow 1:1 atrioventricular nodal conduction likely was

\section{References}

${ }^{1}$ Sawhney NS, Feld GK. Diagnosis and management of typical atrial flutter. Med Clin North Am 2008; 92(1):65-85, x. PMID: 18060998.

${ }^{2}$ Pürerfellner H. Recent developments in cardiovascular drug therapy: Treatment of atrial arrhythmias with new class III drugs and beyond. Curr Med Chem Cardiovasc Hematol Agents 2004; 2(1):79-91. PMID: 15320809.

${ }^{3}$ Camm AJ, Garratt CJ. Adenosine and supraventricular tachycardia. New Engl J Med 1991; 325(23):1621-1629. PMID: 1944450.

${ }^{4}$ Biaggoni I, Killian TJ, Mosqueda-Garcia R, Roberson RM, Robertson D. Adenosine increases sympathetic nerve traffic in humans. Circulation 1991; 83(5):16681675. PMID: 2022024.

5 Rankin AC, Oldroyd KG, Chong E, Rae AE, Cobbe SM. Value and limitation of adenosine in the diagnosis and treatment of narrow and broad complex related to a combination of a relatively slow flutter rate (because of previous antiarrhythmic therapy), an unusually short AV nodal refractory period in the baseline state, and the use of a relatively high dose of adenosine. ${ }^{8}$

\section{Conclusion}

Our case suggested a possible role of adenosine in the rhythm control. The current medications (i.e., amiodarone, procainamide, and sotalol) used for this purpose require multiple administrations and have a wide range of toxicities making the possibility of using a single intravenous dose of adenosine an excellent alternative. Further studies are required before recommending adenosine, except as a last resort when more standard treatment has failed.

tachycardias. Br Heart J 1989; 62(3):195203. PMID: 2789911.

${ }^{6}$ Haines DE, DiMarco JP. Sustained intraatrial reentrant tachycardia: Clinical, electrocardiographic and electrophysiologic characteristics and long-term followup. J Am Coll Cardiol 1990; 15(6):13451354. PMID: 2329238.

7 Garratt CJ, Griffith MJ, O'Nunain S, Ward DE, Camm AJ. The effect of intravenous adenosine on antegrade refractoriness of accessory atrioventricular connections. Circulation 1991; 84(5):1962-1968. PMID: 19343710.

8 Nunain SO, Garratt C, Paul V, Debbas N, Ward DE, Camm AJ. Effect of intravenous adenosine on human atrial and ventricular repolarisation. Cardiovasc Res 1992; 26(10):939-943. PMID: 1486587.

Keywords: atrial flutter, cardiac arrhythmias, adenosine 